\title{
Bioinformatics sequence analysis CD59 stem cells protein
}

\author{
Nawras.T.Jassim ${ }^{* 1}$, S. Ramachandran ${ }^{2}$, M.AfsharAlam ${ }^{3}$. \\ ${ }^{l}$ M.Tech.bioinformatics /Jamiahamdard, India, ${ }^{*}$ chemical engineering /Basra university /Iraq. \\ ${ }^{2}$ CSIR-Institute and Integrative biology/Delhi University/India. \\ ${ }^{3}$ ComputerScience /JamiaHamdard /India
}

\begin{abstract}
Bioinformatics software have been used in the analysis of sequences protein CD59 of stem cells. Using these algorithm in combination with protein CD59 and immune information algorithms lead to better prediction of the destiny of transplant stem cells. This knowledge may help improve the efficacy and safety of stem cells used in transplantation. CD59 protein on cell surface of stem cells isolation from Adipose tissue for Orthologs in other species (human, chimpanzee, monkey, baboon, pig ,rabbit, rat) play an important role on rejection or binding with the complement of the immune system. In this search, we find many strong epitopes by software bioinformatics in MHC class I HLA-A02-01, MHC class II HLA-DR B 1010 receptor with CD59 donor these data may help to design new peptide with active site binding to immune system complement receptor from CD59 donor after isolation from adipose tissue .
\end{abstract}

Keywords: Orthologs, CD59, HLA, MHC

\section{Introduction}

Adult stem cells treatment have been in need for many years in successful treatment of leukemia and related bone or blood cancer using bone marrow transplants [1]. However during transplantation of adult stem cells, immune system tends to reject the transplant due to lack of compatibility [2].In the last few years, scientists have used the human gene associated with CD59 in xenotransplants (animal organs used human transplants). One concern with transplants is that the host cells will reject the new cells, especially when a different species' organs are transplanted. One way to help in the acceptors of an animal's organ is to use a transgenic pig or baboon with the human form of CD59, along with CD46, CD55, and DAF. By using these complement-inhibitory proteins, the human complement system will avoid damaging the pig organs and accept the new organ as its own [3]. Transgenic pigs for the previously listed proteins are necessary for xenotransplantation or the human will undergo rejection and could suffer death [3]. Decay accelerating factor (DAF, CD55) and membrane cofactor protein (MCP, CD46) control the function of C3 convertase [4].In addition, the $20 \mathrm{kDa}$ homologous restriction factor (HRF20, CD59) inhibits assembly of complete large MACs within the membrane of a cell under attack [5-6].

The CD59 protein is a complement system regulator that can inhibit the formation of the membrane attack complex (MAC). It has also been suggested that a less common function of CD59 can influence the proliferation capacity of $\mathrm{T}$ cells and their ability to produce cytokines, which can influence the response of $\mathrm{T}$ cells to a given antigen that may enter the bloodstream. CD59 works as part of the innate immune system, but has also been implicated in the adaptive immune system [4].

CD59 is responsible for inhibiting the C5b-9 complex formation. Consequently the ion channel does not form, the cell does not become lysed, and host cells will continue to live. According to [6].it has been suggested through experimentation that CD59 specifically binds only to C9 through its active site, which later inhibits the pore formation of the MAC[10]. The active site is suspected to be located in the vicinity of a hydrophobic groove from amino acid 16-57 and this section has been found to be the most conserved area of the protein and a mutation in this area can disrupt its function [7].

MHC (genomic region (called HLA, in short for human leukocyte antigen)) are inherited as a group (haplotype), one from each parent. Thus, a heterozygous human inherits one paternal and one maternal haplotype, each containing three class-I (B, C and A) and three class II (DP, DQ and DR) loci. A heterozygous individual will inherit a maximum of 6 class I specificities similarly, the individual will also inherit DP and DQ genes and express both parental antigens. Because the class II MHC molecule consists of two chains (alpha and beta), with some antigenic determinants (specificities) on each chain, and DR alpha- and beta-chains can associate in eithercis helices(both from the same parent) or trans helices (one from each parent) combinations, an individual can have additional DR specificities (Figure 2). Also, there are more than one functional DR betachain genes (not shown in the figure). Hence, many DR specificities can be found in any one individual. [8-9] The immune system normally keeps us healthy by destroying anything in the body it sees as foreign, such as bacteria or viruses. A working immune system recognizes cells from other people as foreign, too. This 
characteristic becomes very important in an allogeneic stem cell transplant (When the stem cells come from another person, it is called an allogeneic transplant). [8]

If the tissue type match between donor and recipient is not close, the patient's immune system may see the new stem cells as foreign and destroy them. This is called graft rejection, and it can lead to graft failure. This is rare when the donor and recipient are well matched. [8-9]

A secondary active binding site of CD59 to C8 and C9 may be on an under loop spanning amino acid Asn8 to Cys13. They are also conserved residues that appear to show binding, but may not be required for its inhibitory function [7]. Finding the active site for CD59 can be beneficial to further research and design of the CD59 inhibitor to possibly block the CD59 active site so lysis may be enhanced on tumor cells, for example [7].

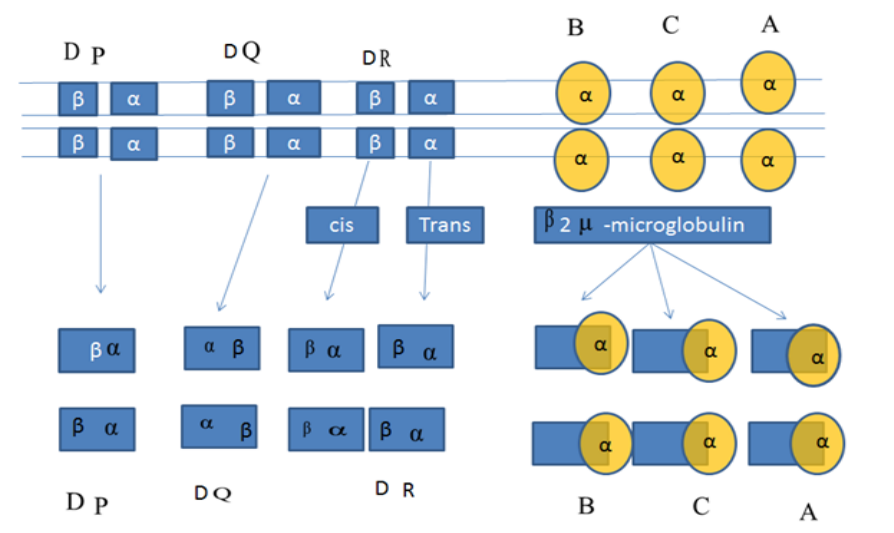

Figure 1: MHC antigens and genes for HLA

\section{Source data}

\section{Method and Material}

Protein sequences of CD59 related surface cell protein was retrieved from National center for Biotechnology Information (http/ncbi/nlm/nih.gov).For simplicity a sequence ID code was given to each molecule multiple sequence alignment was carried out with clustal W program [11]. Table [1] described data set was created by selecting different species.

\begin{tabular}{|l|l|l|l|}
\hline Name of protein & $\begin{array}{l}\text { Protein ID } \\
\text { GI no }\end{array}$ & Species & Length sequence \\
\hline CD59 & GI:49456405 & Homo sapiens (human) & 128 \\
\hline CD59 & GI: 525344037 & Pan troglodytes (chimpanzee) & 144 \\
\hline CD59 & GI:545687592 & Macacafascicularis (monkey ) & 128 \\
\hline CD59 & GI: 402893874 & Papioanubis (olive baboon) & 128 \\
\hline CD59 & GI: 3046390 & Sus scrofa (pig) & 123 \\
\hline CD59 & GI: 130487657 & Oryctolaguscuniculus (rabbit) & 126 \\
\hline CD59 & GI: 1199655 & Rattusnorvegicus (Norway rat) & 124 \\
\hline
\end{tabular}

Aligned sequences sets were represented in Clustal Sequence alignment sequence logos were generated using web(www.ebi.ac.uk) to go application which helps displays the sequence conservation and relative frequencies of the amino acid residues of each position . The CD59 protein conserved pattern of amino acid residues in CD59 protein Orthologous to human. Protein were sequence used MEGA 6.0 version phylogenetic tree was -constructed using neighbor joining method implemented in MEGA version 6.0.We are used Net MHC 3.4 class I [12].predicts binding of peptides CD59 to a number of different HLA alleles (A,B,C) and Net MHC 2.2 server[]. For HLA alleles ( DR,DQ,DP). The Prediction values are given in nMLC50 value (mittlereinhibitors he Konzentration .Next step we are using hydropathy plot is determined using program (http//gcat.davidson, edu/DGPB/KD/KYte-doolittle.htm).hydropaths plot is analysis CD59 protein shows figure(2) two putative transmembrance hydrophobic region with a large intervening hydrophilic domain. 


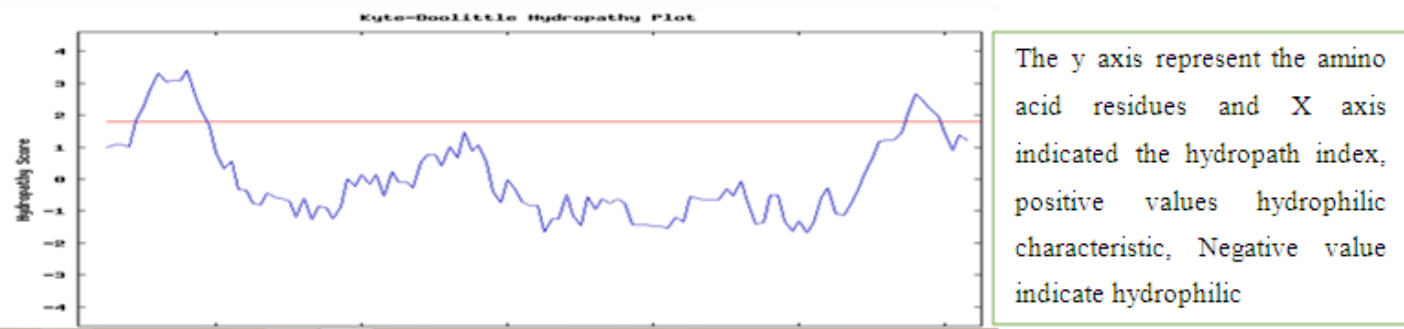

Figure (2): hydropaths plot is analysis CD59 protein shows two putative transmembrane hydrophobic region with a large intervening hydrophilic domain.

\section{Result and discussion}

The proteins sequence taken from Genbank enter with name CD59 protein, complete genome and Accession No and length sequences. This Table [1] is consisted all the database.Theproteins A multiple alignment of CD59 analyzed in this study Orthologs (Human, Chimpanzee, Monkey, Baboon, Pig, Rabbit, Rat) each phylogenetic cluster (Fig.3).

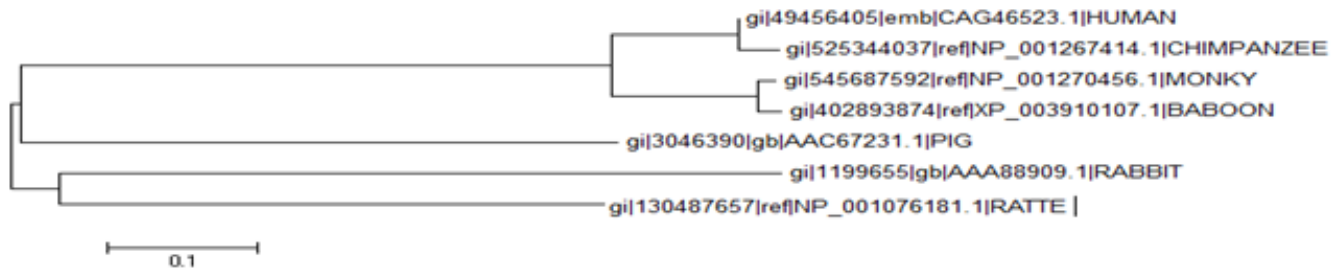

Figure (3): The sequence analysis CD59 proteins result for Orthologs phylogenetic.

\section{Analysis of the conserved regions by MEGA software.}

In this work, various CD59 protein sequence had amino acid lengths between 123-144 amino acids. The multiple sequence alignment of protein sequences show variable sites as well as conserved sites across different species (figure 4 ).First residue of all sequence is Met ( hydrophobic amino acid ) which is conserved in all peptides .In terms of polarity $\mathrm{C}, \mathrm{L}, \mathrm{V}, \mathrm{A}, \mathrm{M}$ are hydrophobic in nature and they can placement in all Orthologous ,after use MEGA software ,we saw that the residues $\mathrm{C}$ and $\mathrm{L}$ have conserved position in 10 site Figure (5) and the $\mathrm{A}$ and $\mathrm{V}$ residues are conserved in two position and $\mathrm{M}$ is conserved only in one position.

These conserved residues help to create the signature residues of new epitope domain .In other resulted orthology protein, substitutions of amino acids of CD59 are slightly low in number CD59 protein is observed substitution of amino acid in terms of polarity in from negative (hydrophilic) amino acid S, T, D, Y,N.Finally in these sequence we found that the amount of positively charged amino acid residues are more than negative charged residues .

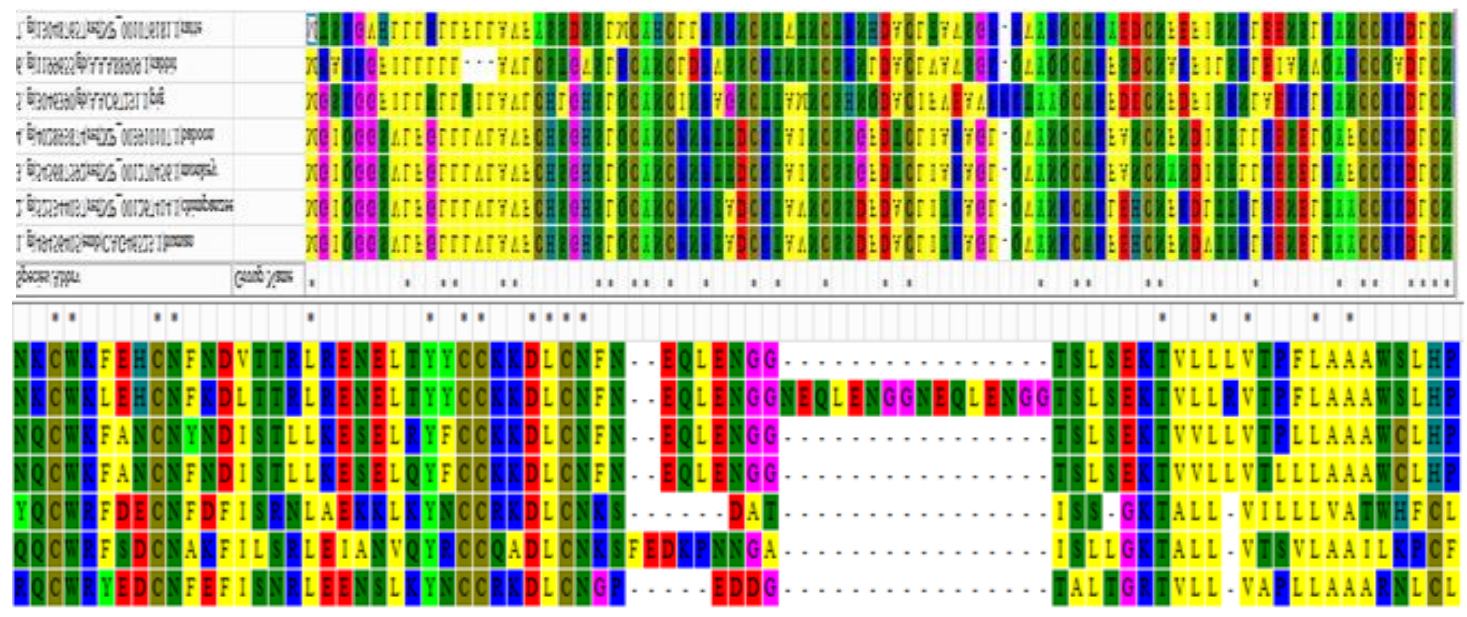

Figure 4: multiples sequences alignment of MAGA software various CD59proteins, in terms of polarity amino acid for sequences CD59 were $(\mathrm{C}, \mathrm{L}, \mathrm{V}, \mathrm{A}, \mathrm{W})$ the placement of residues in CD59 data base of topology. 


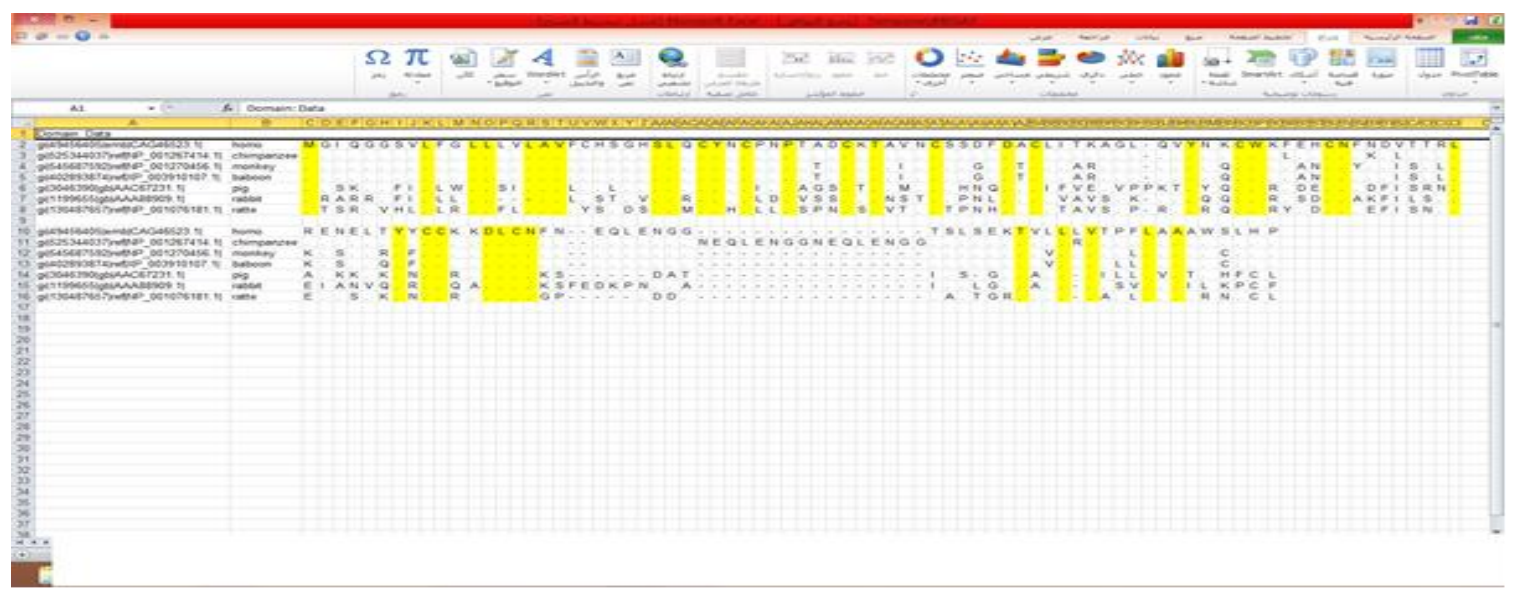

Figure 5: Amino acid conserved position.

\section{MHC the major histocompatibility complex}

After we selected the common sequence between different species by MEGA, we have used MHC software for finding the strong binding sit of these sequence. The MHC I given us these results, three peptides of 9 residues have the position between 1 to 44 on CD59 differing by one residue.

These 3 peptides have strong bindings include of SVLFGLLLV, VLFGLLLVL and SLSEKTVLL in positions 6, 7and 105 of CD59, and these peptides have this HLA-A02-01 allele structure of MHC I. However pervious study show the active site of CD59 between 1 to 77 . Finally the position 6 and 7 are strong binding in CD59 with inhibition immune system. The weak binding sit in human and orthology is the position 110 for thealleleconsciencesHLA-A02:01 for class I (ALLVTSVLA).

As well as, the MHC class II the position 47, 48, 49, 50 have strong binding for (DFDALIIKAGLQUY) (DFDACLITKAGLQV) (DACLITKAGCQUYNK) (ACLIIKAGCQUYNKC) for HLA-DRB10101 of all species. The weak bindings in all different species are between 1-10 in class II for HLADRB10101 with peptide (MGIQGGSVLFGLLLV).

\section{Kyte Doolittle Hydropathy Plot:}

We have used hydropathy plot for epitope strong binding for check it's hydrophilic or hydrophobic.We saw the MHC class I epitope (SVLFGLLLV) and (VLFGLLLVL) is depend on the figure (6) the epitope present in charge positive (hydrophobic).

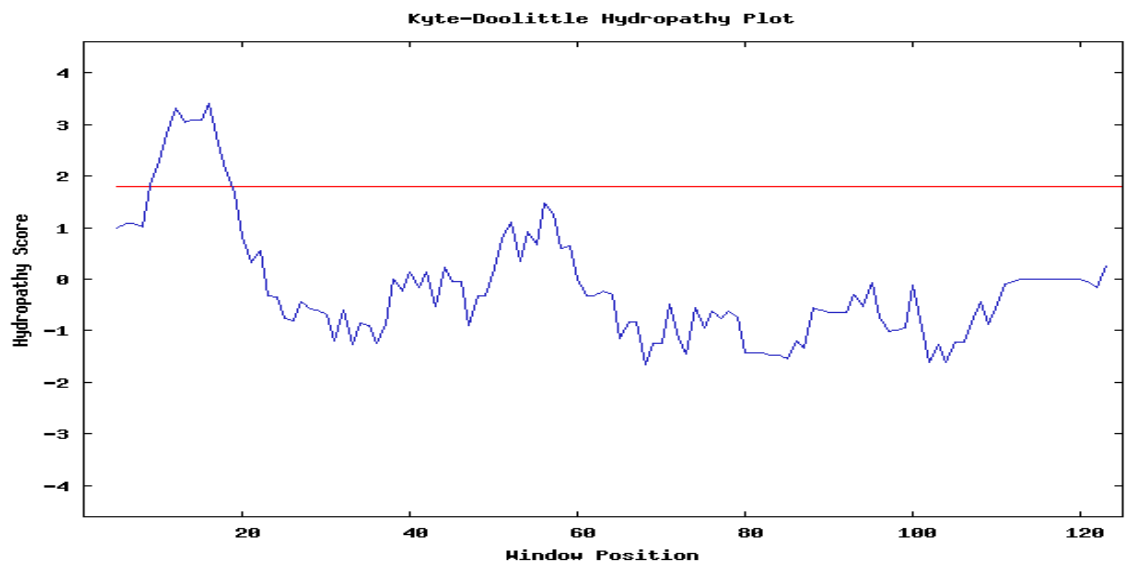

Figure (6) explain the strong binding of hydrophobic region, the positive charge (hydrophobic) and strong binding of epitope. 


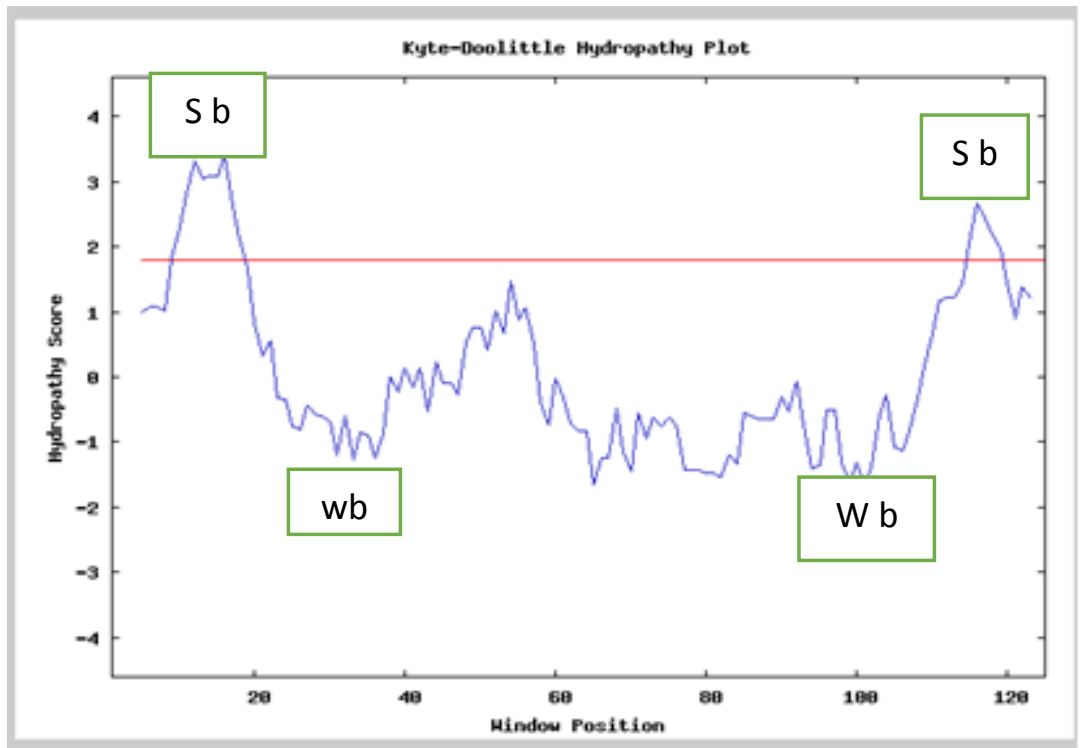

Figure 7: peptide of 8 residues on 6and 7 of SVLFGLLLV and VLFGLLLVL sequence CD59 for and HLAA02-01 structure of MHC I on hydrophilic strong site.

$* \mathrm{~W} \mathrm{~b}=$ weak binding

$* \mathrm{~S} \mathrm{~b}=$ strong binding

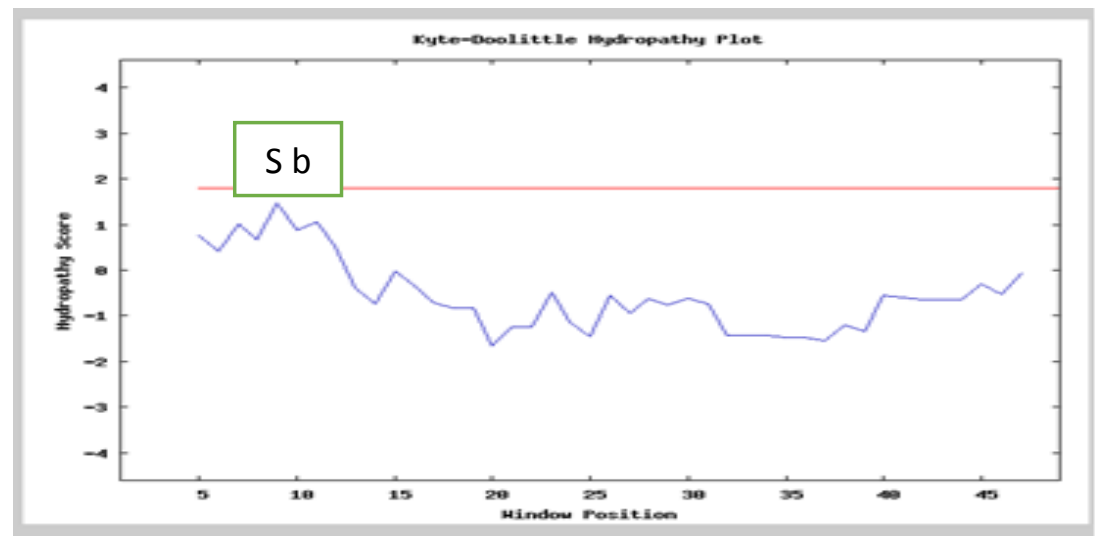

Figure 8: peptide of 15 residues on 48of FDACLITKAGLQVYN sequence CD59 for and HLA -DR -B1010 structure of MHC II on hydrophilic strong site.

They are many proteins to active of complement system during the terminal complement pathgene surface, C3 protein after activity is become hydrophilic cell [14]. C9 has two active sides, $\mathbf{a}$ and $\mathbf{b}$. The side of $\mathbf{a}$ is hydrophilic and $\mathbf{b}$ is hydrophobic with along complement components (C5b, C6, C7, C8) figure (9). The compounds are become.hydrophobic and attach with C9a (hydrophilic), in our work when analysis CD59 epitope strong binding with MHC CLASS I and CLASS II, We received many epitope hydrophobic of CD59. We hope to find out a new epitope hydrophobic which binding to $\mathrm{C} 3$ and $\mathrm{C} 9$ to inhibit immune system. 


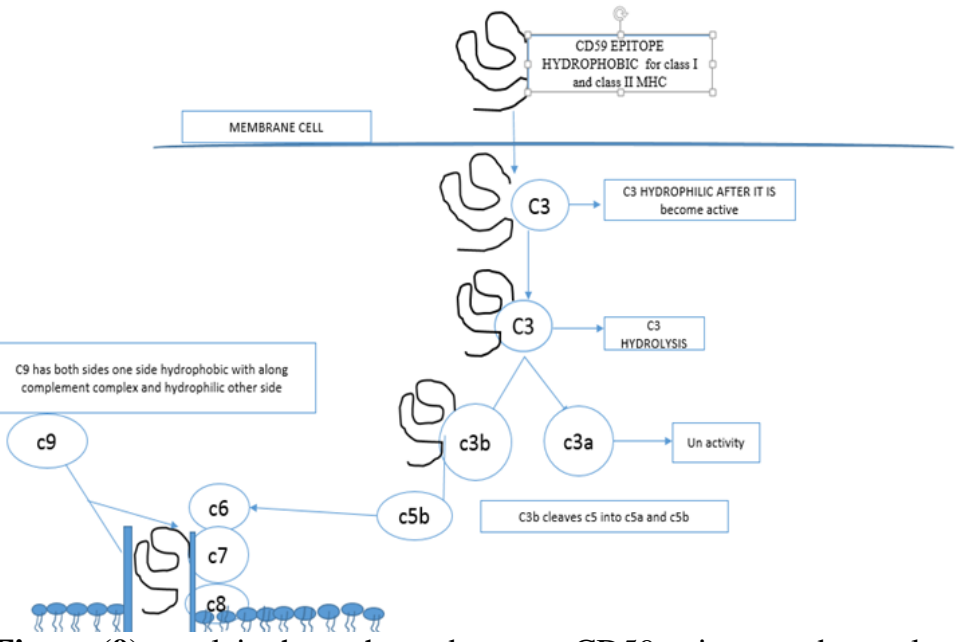

Figure (9): explain the pathway between CD59 epitope and complement system.

\section{Conclusion}

The aim of this study to carry out bioinformatics analysis stem cell .with specific reference to the immune system .Stem cell transplants can use cells from your own body (autologous stem cell transplant), from donor (allogeneic stem cell transplant) or from an identical twin (syngeneic transplant)A stem cell transplant also may be performed if high-dose chemotherapy or radiation.We will fine new way between different family.CD59 is recognize the adult stem cells The function of protein CD59 on cell surface of stem cells different species plays an important role in rejection or binding with complement the immune system .During my work we find many strong binging epitopes between CD59 and the immune system by sequence analysis and week binging epitope using bioinformatics. In future. When the analysis of CD59 protein stem cell from adipose tissue was distributed between MHC I on the allele AHL -A02-01 where MH class II was on allele HALDR -B -1010 in hydrophobic. However the difficulty of obtaining the software bioinformatics because very expensive and the stem cells in order to provide isolation these techniques lab orations if limited .may hinder the development of this work. In future is lead to prepare new peptide withe active site binding to immune system complement receptor from CD59 donor after isolation from adipose tissue stem cells and also after prepare new peptide any receptor is not need searching much tissue we will going dictate to bank stem cells

\section{Acknowledgements}

First of all, I would like to thank my advisor Professor Mr.M. Afshar Alam, Professor, Department of Computer Science, Jamia Hamdard, New Delhi. For his guidance through my entire master process .From the beginning when I first entered Delhi and knew little about the field and I would also like to thank my co-guide Dr .S.Ramachandran, CSIR-Institute of Genome and Integrative Biology

\section{Reference}

[1]. National Library of Medicine (www.nlm.nih.gov/medlineplus/healthtopics.html).

[2]. Galili, U (Dec 2005). "The alpha-gal epitope and the anti-Gal antibody in xenotransplantation and in cancer immunotherapy". Immunology and cell biology 83 (6): 674-86. doi:10.1111/j.1440-1711.2005.01366.x. PMID 16266320.

[3]. Lambrigts, Denis; Sachs, David H.; Cooper, David K.C. 15 Sept 1998. Discordant Organ Xenotransplantation in Primates: World Experience and Current Status. Transplantation. 66(5): 547-561.

[4]. Longhi, M. Paula; Harris, Claire L.; Morgan, B. Paul; Gallimore, Awen. Feb 2006. Holding T cells in check - a new role for complement regulators? Trends in Immunology. 27(2):102-108

[5]. DAVIES,A.,SIMMONS,D.L.,HARRISON,R.A.,TIGHE,H.,LACHMANNP.J.\&WALDMANN，H. (1989). CD59, an Ly-6-like protein expressed in human lymphoid cells, regulates the action of the complement membrane attack complex on homologous cells.Journal of Experimental Medicine170, 637-654.

[6]. McKusick, Victor A. 9 Oct 2001. CD59 Antigen, Protectin. Online Mendelian Inheritance in Man (OMIM). Accessed 9 Mar 2006 http://www.ncbi.nlm.nih.gov/entrez/query.fcgi?db=OMIM.

[7]. Yu, By Jinghua; Abagyan, Ruben; Dong, Shanghong; Gilbert, Alexander; Nussenzweig, Victor; Tomlinson, Stephen. 17 Feb 1997. Mapping the Active Site of CD59.Journal of Experimental Medicine. 185(4): 745-753.

[8]. Role of HLA in Stem Cell Transplantation. Last Medical Review: 10/02/2013.

[9]. Britta Eiz-Vesper and Rainer Blasczyk Institute for Transfusion Medicine, Hannover Medical School, Hannover Germany Relevance of HLA Expression Variants in Stem Cell Transplantation.

[10]. S Le Bas-Bernardet1, I Anegon1 and G Blancho1, 2, Progress and prospects: genetic engineering in xenotransplantation, Gene Therapy (2008) 15, 1247-1256; doi:10.1038/gt.2008.119. 
[11]. Thompson JD, et al. Nucleic Acids Res. 1997; 25:4876. [PMC free article] [PubMed].

[12]. Center for Biological Sequence Analysis - CBS, Department of Systems Biology, The Technical University of Denmark - DTU, Kemitorvet Build. 208, 2800 Lyngby, Denmark. Accurate approximation method for prediction of class I MHC affinities for peptides of length 8,10 and 11 using prediction tools trained on 9mers, Oxford JournalsLife Sciences \& Mathematics \& Physical Sciences Bioinformatics Volume 24, Issue 11Pp. 1397-1398.

[13]. 1Center for Biological Sequence Analysis, Department of Systems Biology, Technical University of Denmark, Building 208, DK2800 Lyngby, Denmark. NN-align.An artificial neural network-based alignment algorithm for MHC class II peptide binding prediction, BMC Bioinformaticsv.10; 2009PMC2753847.Book:

[14]. Janeway CA Jr, Travers P, Walport M, et al. Immunobiology: The Immune System in Health and Disease. 5th edition. New York: Garland Science; 2001.book 